\begin{tabular}{ll}
\hline \hline MINING AND METALLURGY INSTITUTE BOR & ISSN: 2334-8836 (Štampano izdanje) \\
UDK: 622 & ISSN: 2406-1395 (Online) \\
\hline \hline
\end{tabular}

\title{
HARMONICS ASYNCHRONOUS TORQUES IN WOUND ROTOR INDUCTION MOTOR WITH FREQUENCY CONVERTER IN THE ROTOR
}

\begin{abstract}
The subject of this paper is the harmonics asynchronous torqueses which appear due to the presence of high time harmonics in the voltage and current of wound rotor induction motor whose speed is regulated by frequency converter in the rotor circuit. Theoretical and experimental analysis was carried out in the case when the waveform of voltage, obtained from frequency converter, is rectangular.

Keywords: harmonics asynchronous torques, induction motor, high harmonics, belt-drive
\end{abstract}

\section{INTRODUCTION}

In various branches of the industry, where bulk materials are produced or transported, various types of belt conveyors, with various constructions and drive systems, are used for transport of materials. A characteristic place for application the belt conveyors is mines, especially the open-pit mines. For the operation of belt conveyors, the most important component is the belt drive. Belt conveyors which are used for transport of bulk materials most often have a large capacity, which means driving them requires drives with significant power. With belt conveyors, it is especially necessary to highlight the need for a high torque during starting the belt, i.e., a high starting torque. A drive with wound rotor induction motor is often used for belt-drive solution at the open-pit mines [1].

In the case when the speed of wound rotor induction motor is regulated by frequency converter in the rotor circuit, in the stator and rotor voltages and currents, in addition to the fundamental harmonic, the high time harmonics appear as well. The spectrum of high time harmonics depends on the type of used converter, motor construction and mode of drive operation. High harmonics have a negative influence on regulated motor characteristics. This negative influence is manifested in the appearance of additional iron and copper loss and asynchronous torques [2, 5].

\footnotetext{
* Faculty of Agriculture, University of Belgrade, Serbia

** University of Priština, Faculty of Technical Sciences, Kosovska Mitrovica, Kneza Miloša 7, 38220 Kosovska Mitrovica, Serbia, e-mail: statkic@etf.rs

**** These results are a part of the Project TR 33016, "Research, Development and Implementation of Programs and Procedures Energy Efficiency of Electric Drives" funded by the Ministry of Education, Science and Technological Development of the Republic of Serbia.
} 


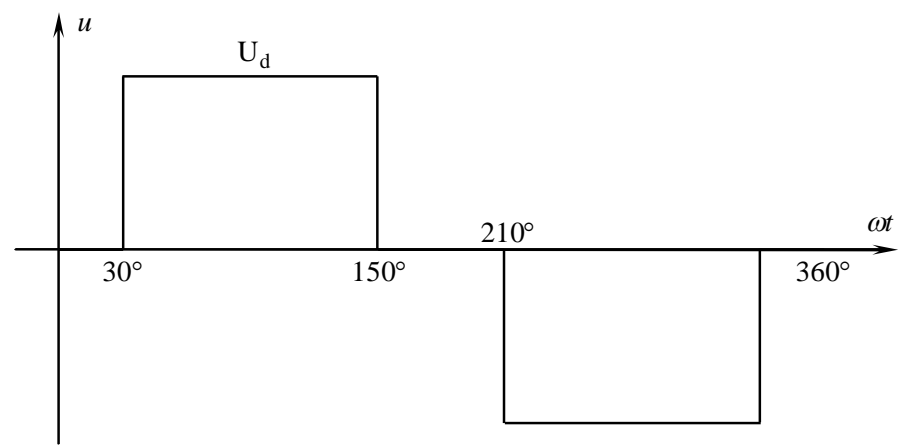

Figure 1 The rectangular waveform of the rotor phase voltage

Asynchronous harmonic torques, which are analyzed in this paper, are produced by interaction of rotor flux high time harmonics and corresponding stator current harmonics in the case when voltage static converter is used. The rectangular voltage waveform, controlled by the frequency converter in rotor circuit, is shown in Figure 1.

Periodic voltage waveform shown in Figure 1, in addition to the fundamental, consists of high harmonics in order $v=5,7,11$, $13, \ldots$ as well. Due to the presence of high harmonics in the rotor voltage, the rotor and stator currents are not sinusoidal, i.e. in addition to the fundamental, high harmonics appear. Frequencies of these harmonics are given by the following equations:

$$
\begin{aligned}
& f_{1_{v}}=[1-(1 \pm v) s] f_{1} \\
& f_{2_{v}}=s v f_{1}
\end{aligned}
$$

With symbol $s$ is marked a slip, while the $f_{1}$ represents the network frequency at the stator side.

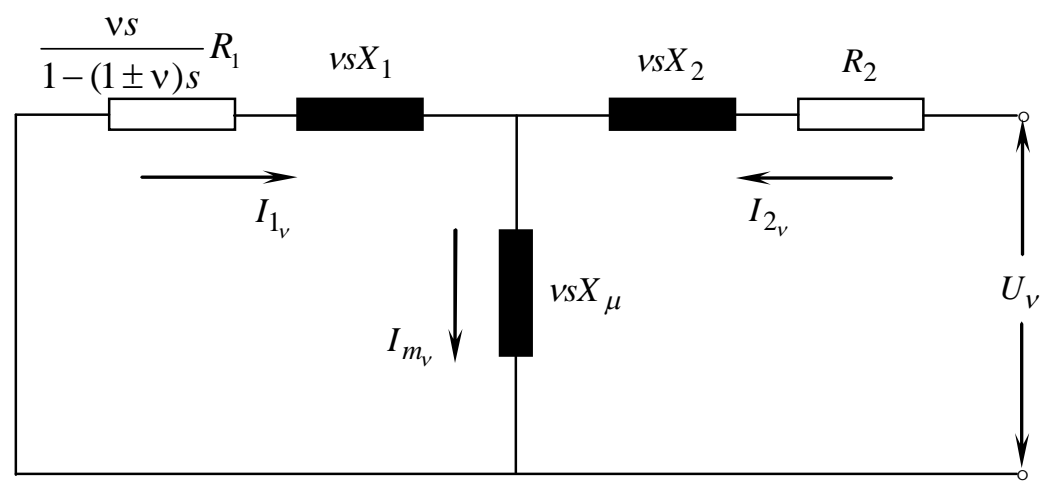

Figure 2 Equivalent circuit of wounded rotor induction motor for high time harmonics

High voltages and currents time harmonics in the stator and rotor circuits, as well as asynchronous harmonic torques, can be analyzed and calculated according to the equivalent circuit of wounded rotor induction motor for high harmonics [4], shown in Figure 2. Symbol designations used in the equivalent circuit are taken from the basic theory of induction motors. 


\section{GENERAL EQUATIONS}

Harmonic asynchronous torque of $v^{\text {th }}$ high time harmonic is:

$$
M_{v}=\frac{q}{\Omega_{1_{v}}} \frac{R_{1}}{s_{v}} I_{1_{v}}^{2}
$$

For the vth harmonic of the stator current speed of the rotating field is $\Omega_{1 v}=\mathrm{vs} \Omega_{1}$, while slips is given with the expression $s_{v}=\frac{1-(1 \pm v) s}{v s}$ [4]. According to equivalent circuit which is shown in Figure 2, the $v^{\text {th }}$ harmonic of the stator current can be expressed through the $v^{\text {th }}$ harmonic of the rotor current.

$$
I_{1_{v}}=I_{2_{v}} \frac{X_{\mu_{v}}}{\sqrt{\left[\frac{R_{1}}{1-(1 \pm v) s}\right]^{2}+\left(X_{1}+X_{\mu_{v}}\right)^{2}}}
$$

where is $v=5,7,11,13, \ldots$ the harmonic order.

Substitution equation (3) in the equation (2), expression for harmonic asynchronous torque of the $v$ th high time harmonic receives the following form:

$$
M_{v}=\frac{q}{\Omega_{1}} \frac{R_{1}}{1-(1 \pm v) s} I_{2_{v}}^{2} \frac{X_{\mu_{v}}^{2}}{\left[\frac{R_{1}}{1-(1 \pm v) s}\right]^{2}+\left(X_{1}+X_{\mu_{v}}\right)^{2}}
$$

Relative value of the harmonic asynchro- nous torque of vth high time harmonic is:

$$
m_{v}=\frac{M_{v}}{M_{1}}=\left(\frac{I_{2_{v}}}{I_{2_{1}}}\right)^{2} \frac{s R_{1} X_{\mu_{v}}^{2}}{R_{2}[1-(1 \pm v) s]\left\{\left[\frac{R_{1}}{1-(1 \pm v) s}\right]^{2}+\left(X_{1}+X_{\mu_{v}}\right)^{2}\right\}}
$$

Maximum value, hence most imtorque of the $5^{\text {th }}$ harmonic, which according portant significance, has the asynchronous to exp. (5) is:

$$
m_{5}=\left(\frac{I_{25}}{I_{2}}\right)^{2} \frac{s R_{1} X_{\mu_{5}}^{2}}{R_{2}(1-6 s)\left[\left(\frac{R_{1}}{1-6 s}\right)^{2}+\left(X_{1}+X_{\mu_{5}}\right)^{2}\right]}
$$

Maximum value of the asynchronous torque of the $5^{\text {th }}$ harmonic is for $s=1 / 6$. In order to estimate value of this torque we are taking that $s=1 / 6-\Delta s$. Considering that $\Delta \mathrm{s}<<1 / 6$, it is obtained that:

$$
m_{5 \max }=\left(\frac{I_{2_{5}}}{I_{2_{1}}}\right)^{2} \frac{X_{\mu_{5}}^{2}}{12 R_{2}\left(X_{\mu_{5}}+X_{1}\right)^{2}}
$$




\section{THEORETICAL ANALYSIS}

From the expression (5), it can be seen that asynchronous high harmonic torques value depends on a sort of the used static semi-conductor frequency converter and the order of high time harmonic, where with the increase of the order of harmonic, that value rapidly decreases. For the given value of higher harmonic, character and the value of higher harmonics asynchronous torques depend on the slip, i.e. of the rotation speed. So, the $5^{\text {th }}$ harmonic torque is resistive in the region $0<s<1 / 6$, i.e. for speed in the interval $5 / 6 n_{1}<n<n_{1}$ while it is driven one in the region $1 / 6<s<1$, i.e. for speed $0<n<5 / 6 n_{1}$. The torque of the $7^{\text {th }}$ harmonic is resistive at all speeds. The torque of the $11^{\text {th }}$ harmonic is driven in the region $0<n<11 / 12 n_{1}$ and resistive one afterwards, etc.

According to the expressions (6) and (7), it could be concluded that maximum value of the $5^{\text {th }}$ harmonic asynchronous torque, appearing at $s=1 / 6$ is much higher than at the other values and that it has high relative value. Actually, it was stated in some previously published studies. However, that is not so, owing to the following reasons:

Firstly, reactance $X_{\mu_{5}}$ is lower than corresponding reactance for fundamental harmonic $X_{\mu_{1}}\left(X_{\mu_{5}}<X_{\mu_{1}}\right)$, because of saturation due to the field fundamental harmonic [3].

Secondly, at the slip of $s=1 / 65^{\text {th }}$ harmonic of the stator current is equal zero $\left(I_{15}=0\right)$, because then the flux of that harmonic is highly increased, as there is no damping effect of stator current. This results in a significant increase of corresponding equivalent reactance, and directly comes from equivalent circuits for the $5^{\text {th }}$ harmonic at $s=1 / 6$ and at $s \neq 1 / 6$, shown in Figures 3 and 4.

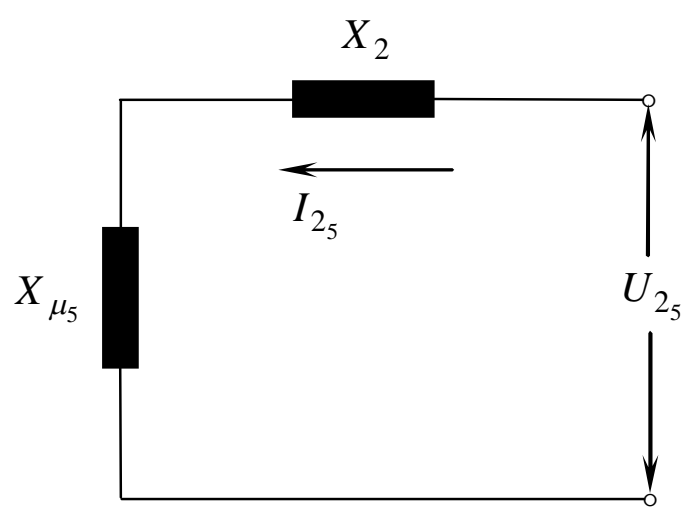

Figure 3 Equivalent circuit of wounded rotor induction motor for the $5^{\text {th }}$ harmonic at $s=1 / 6$

Consequently, the $5^{\text {th }}$ harmonic of rotor current at $s=1 / 6$ is lower than at $s \neq 1 / 6$ because:

$$
\frac{I_{2_{5(s \approx 1 / 6)}}}{I_{2_{5(s \neq 1 / 6)}}}=\frac{X_{2}\left(1+c_{v_{5}}\right)}{c_{v_{5}}\left(X_{2}+X_{\mu_{5}}\right)}
$$




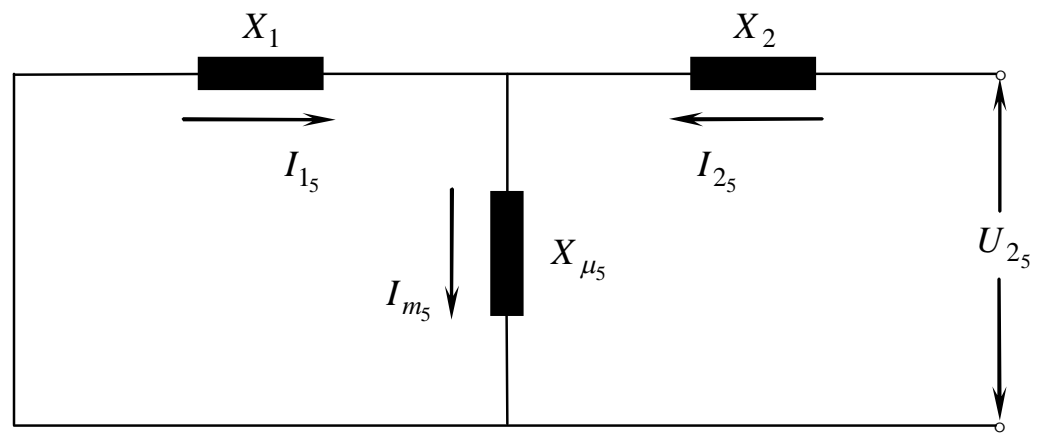

Figure 4 Equivalent circuit of wounded rotor induction motor for the $5^{\text {th }}$ harmonic at $\mathrm{s} \neq 1 / 6$

Where $c_{v_{5}}=\frac{X_{1}+X_{\mu_{5}}}{X_{\mu_{5}}}$ is the Hop-

kins leakage coefficient of the $5^{\text {th }}$ harmonic which amounts $c_{v_{5}}=1.15-1.3$ for recent induction motors, and $X_{\mu 5}=(3-6) X_{2}$, i.e.:

$$
I_{25(s=1 / 6)}<I_{25(s \neq 1 / 6)}
$$

Since the $5^{\text {th }}$ harmonic of rotor current has a ignificant effect on maximum value of corresponding harmonic asynchronous torque (according to expression (8)), it can be concluded that this value is much lower than it would be if the facts, mentioned above, were neglected.

Asynchronous harmonic torque of the 7th harmonic has no maximum in motor working domain. The maximum value of the $11^{\text {th }}$ harmonic appears at the slip $s=1 / 12$ and it is much lower than maximum value of the $5^{\text {th }}$ harmonic torque.

\section{RESULTS OF MEASUREMENTS AND CALCULATIONS}

In order to verify the derived conclusions and insight into relative values of the asynchronous harmonic torques experimental tests have been carried out for three-phase wound rotor induction motor made by "Rade Končar", of $6.4 \mathrm{~kW}$ rated output, which parameters are determined by previous experimental tests and which amount:

$$
\begin{array}{ll}
R_{1}=0.41 \Omega & R_{2}=0.60 \Omega \\
X_{1}=1.25 \Omega & X_{2}=1.25 \Omega \\
X_{\mu_{5}}=4.6 \Omega &
\end{array}
$$

Feeding from rotor's side was through semi-conductor converter type VF-6 made by GEC, whose output was voltage shape shown in Fig. 1. Measuring of harmonic stator's and rotor's currents was carried out using electronic Wave analyzer FRA$2 \mathrm{~d}$ made by "Radiometar"- Copenhagen. The following results were obtained:

$$
\begin{array}{ll}
\text { - at slip of } s \approx 1 / 6 & I_{25}=11 \% \\
\text { - at slip of } s=0,5 & I_{25}=28 \%
\end{array}
$$

By calculation, according to (8), maximum value of the asynchronous torque of $5^{\text {th }}$ harmonic is obtained:

$$
m_{5 \max }=0,81 \%
$$

For medium and high power motors, relative value of stator resistance is very low, because higher value of $m$ is obtained which, however do not exceed the value of $5 \%$. Value of torque at other slips is lower than $1 \%$. 


\section{CONCLUSION}

Harmonic asynchronous torques appear due to the presence of high time harmonics in voltages and currents of wound rotor induction motor whose speed is regulated by the static frequency converter in the rotor circuit. They are produced by the interaction between the high time harmonics rotor flux and corresponding stator current harmonics. Theoretical analysis, calculations carried out and experimental test results, which are given in this paper, have shown that they are small, including maximum value of the $5^{\text {th }}$ harmonic torque, which appears at the slip of $s=1 / 6$ and that they have no significant effect on motor behavior.

The described method is considered the most unfavorable voltage waveform at the output of the frequency converter, and it is a rectangular voltage waveform, and showed that the asynchronous torques cannot have a significant impact on the value of the motor torque. Further shaping the voltage waveform can completely eliminate the presence of asynchronous components of the motor torque.

\section{Appendix}

$s \quad$ slip

$\Omega_{1} \quad$ speed of stator rotated field

$f_{1} \quad$ network frequency at the stator's side

$f_{2} \quad$ frequency at the rotor's side

$v \quad$ ordinal number of harmonics

$\Omega_{1 v}$ speed of the vth harmonic of stator rotated field

$s_{v} \quad$ slip of the $v^{\text {th }}$ harmonic

$R_{1} \quad$ stator resistance per phase

$R_{2} \quad$ rotor resistance per phase

$X_{1} \quad$ stator leakage reactance per phase

$X_{2}$ rotor leakage reactance per phase

$X_{\mu} \quad$ magnetization reactance for the base frequency

$X_{\mu \nu}$ magnetization reactance of the $v^{\text {th }}$ harmonic

$U_{1 v}$ stator voltage per phase for the $v^{\text {th }}$ harmonic
$U_{2 v}$ rotor voltage per phase for the $v^{\text {th }}$ harmonic

$I_{1} \quad$ rms stator current for the base frequency

$I_{2} \quad$ rms rotor current for the base frequency

$I_{1 v} \quad$ rms stator current for the vth harmonic

$I_{2 v} \quad$ rms rotor current for the $v$ th harmonic

\section{REFERENCES}

[1] B. Jeftenić, M. Bebić, L. Ristić, S. Štatkić, "Design and Selection of Belt Conveying Equipment \& Systems," Chapter 2 in Book - Design and Selection of Bulk Material Handling Equipment and Systems: Mining, Mineral Processing, Port, Plant and Excavation Engineering. vol. I, Edited by Prof. Jayanta Bhattacharya, ISBN: 9788190904377, pages in Book: 254, pages in Chapter 2: 60, Publisher: Wide Publishing, Kolkata, West Bengal, India, 2011.

[2] A. Lavi, R.J. Polge: Induction Motor Speed Control with Static Inverter in the Rotor, IEEE Trans, PAS-85, No 1, 1987, pp. 76-84.

[3] M. Petrović: The Behavior of Asynchronous Machine in the Regulation Using the Thyristor Recovery of Slip Energy, Publications of Faculty of Electrical Engineering, University of Belgrade, Series Electro Energy, No. 63, 1976 (in Serbian);

[4] Dj. Vukić: Harmonic Equivalent Circuits of Induction Motor with Semiconductor Converter in the Rotor, Review of research work at the Faculty of Agriculture, Vol. 38, 1993, pp. 119-127.

[5] Dj. Vukić: D. Stojanović, N. Mitrović: The Effect of Higher Harmonics on Operation the Asynchronous Motor with Semiconductor Converter in the Rotor Circuit, Proceedings, VIII Symposium "Power Electronics", Novi Sad, September 1995, pp. 343-350 (in Serbian). 


\begin{tabular}{ll}
\hline \hline INSTITUT ZA RUDARSTVO I METALURGIJU BOR & ISSN: 2334-8836 (Štampano izdanje) \\
UDK: 622 & ISSN: 2406-1395 (Online) \\
\hline \hline
\end{tabular}

Đukan Vukić, Saša Štatkić**, Žarko Milkic ***

\section{HARMONIJSKI ASINHRONI MOMENTI ASINHRONOG MOTORA SA NAMOTANIM ROTOROM SA FREKVENTNIM PRETVARAČEM U ROTORU ${ }^{* * *}$}

Izvod

Predmet ovog rada su harmonijski asinhroni momenti koji nastaju kao posledica postojanja vremenskih harmonika napona i struje kod asinhronog motora sa namotanim rotorom kod koga se upravljanje brzinom vrši frekventnim pretvaračem u kolu rotora. Sprovedena je teoretska i eksperimentalna analiza za slučaj kada je talasni oblik napona na izlazu iz frekventnog pretvarača pravougaoni.

Ključne reči: harmonijski asinhroni momenti, asinhroni motor, viši harmonici, pogoni traka

\section{UVOD}

U različitim granama industrije gde se rastresiti materijali proizvode ili prevoze, za transport materijala upotrebljavaju se različiti tipovi transportera sa gumenom trakom, različitih konstrukcija i pogonskih sistema. Karakteristično mesto za primenu transportera sa gumenom trakom su rudnici, posebno rudnici sa površinskim kopom. Za rad transportera sa gumenom takom najvažnija komponenata je pogon trake. Transporteri sa gumenom trakom koji se koriste za prevoz rastresitih materijala često su velikog kapaciteta, što za njihovo pokretanje zahteva pogone značajnih snaga. Kod transportera sa gumenom trakom neophodno je posebno naglasiti potrebu za velikim momentom $\mathrm{u}$ toku polaska trake, tj. velikim polaznim momentom. Pogon sa asinhronim motorom koji ima namotani rotor se često koristi kao rešenje za pogone traka na površinskim kopovima [1].

U slučaju kada se upravljanje brzinom asinhronog motora sa namotanim rotorom vrši pomoću frekventnog regulatora u kolu rotora, pored osnovnih harmonika struja i napona statora i rotora postoje i viši vremenski harmonici. Sadržaj viših vremenskih harmonika zavisi od vrste upotrebljenog pretvarača, konstrukcije motora i režima rada pogona. Viši harmonici imaju negativan uticaj na karakteristike upravljanja motora. Ovaj negativan uticaj ispoljava se u pojavljivanju dodatnih gubitaka u gvožđu i bakru i harmonijskih asinhronih momenata [2, 5].

\footnotetext{
* Poljoprivredni fakultet, Univerzitet u Beogradu, Srbija

*** Fakultet tehničkih nauka, Univerzitet u Prištini, Kosovska Mitrovica, Kneza Miloša 7 , 38220 Kosovska Mitrovica, Srbija, e-mail: statkic@etf.rs

*** Ovi rezultatu su deo projekta TR33016 "Istraživanje, razvoj i primena programa i mera energetske efikasnosti elektromotornih pogona" finansiranog od Ministarstva prosvete, nauke i tehnološkog razvoja Republike Srbije
} 


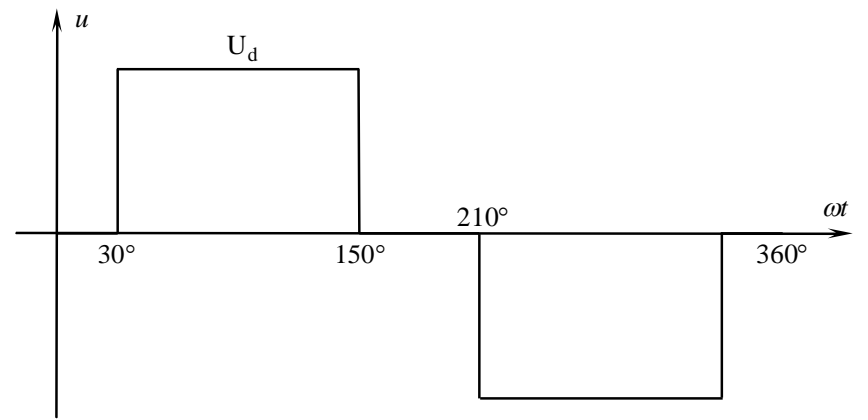

Sl. 1. Pravougaoni talasni oblik faznog napona rotora

Harmonijski asinhroni momenti koji su analizirani u ovom radu, nastaju usled međusobnog delovanja viših vremenskih harmonika fluksa rotora i odgovarajućih harmonika statorskih struja u slučaju kada se koristi statički naponski pretvarač. Pravougaoni talasni oblik napona upravljan od strane frekventnog pretvarača u kolu rotora prikazan je na slici 1.

Periodični talasni oblik napona prikazan na slici 1 , pored osnovne komponente takođe sadrži i više neparne harmonike prema redosledu $v=5,7,11,13, \ldots$. Kao posledica prisustva viših harmonika napona rotora, struje rotora i statora nisu sinusoidalne, tj., pored osnovne sinusne komponente, sadrže i više harmonike. Frekvencije ovih viših harmonika date su sledećim jednačinama:

$$
\begin{aligned}
& f_{1_{v}}=[1-(1 \pm v) s] f_{1} \\
& f_{2_{v}}=s v f_{1}
\end{aligned}
$$

Klizanje je označeno sa simbolom $s$, dok $f_{1}$ predstavlja frekvenciju mrežnog napajanja sa strane statora.

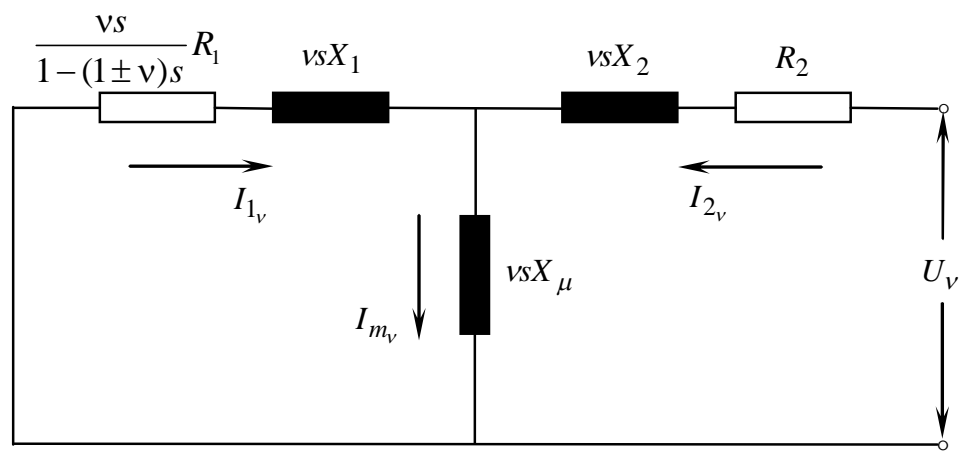

Sl. 2. Ekvivalentna šema asinhronog motora sa namotanim rotorom za više vremenske harmonike

Viši vremenski harmonici napona i struja u kolima statora i rotora, kao i harmonijski asinhroni momenti, mogu se analizirati i izračunavati na osnovu ekvivalentne šeme asinhronog motora sa namo- tanim rotorom za više harmonike [4], koja je prikazana na Slici 2. Primenjeno označavanje simbola u ekvivalentnoj šemi preuzeto je iz osnovne teorije asinhronih motora. 


\section{OPŠTE JEDNAČINE}

Harmonijski asinhroni moment za v-ti viši vremenski harmonik je:

$$
M_{v}=\frac{q}{\Omega_{1_{v}}} \frac{R_{1}}{s_{v}} I_{1_{v}}^{2}
$$

Za $v$-ti harmonik struje statora brzina obrtnog polja statora je $\Omega_{1_{v}}=v s \Omega_{1}$, dok je klizanje dato izrazom $s_{v}=\frac{1-(1 \pm v) s}{v s}$, [3]. Prema ekvivalentnoj šemi koja je prikazana na Slici 2. v-ti harmonik struje sta- tora se može izraziti preko $v$-tog harmonika struje rotora:

$$
I_{1_{v}}=I_{2_{v}} \frac{X_{\mu_{v}}}{\sqrt{\left[\frac{R_{1}}{1-(1 \pm v) s}\right]^{2}+\left(X_{1}+X_{\mu_{v}}\right)^{2}}}
$$

gde je $v=5,7,11,13, \ldots$ red harmonika.

Zamenom jednačine (3) u jednačinu (2) izraz za harmonijski asinhroni moment za $v$-ti viši vremenski harmonik dobija sledeći oblik:

$$
M_{v}=\frac{q}{\Omega_{1}} \frac{R_{1}}{1-(1 \pm v) s} I_{2_{v}}^{2} \frac{X_{\mu_{v}}^{2}}{\left[\frac{R_{1}}{1-(1 \pm v) s}\right]^{2}+\left(X_{1}+X_{\mu_{v}}\right)^{2}}
$$

Relativna vrednost harmonijskog asin- harmonik je: hronog momenta za $v$-ti viši vremenski

$$
m_{v}=\frac{M_{v}}{M_{1}}=\left(\frac{I_{2_{v}}}{I_{2_{1}}}\right)^{2} \frac{s R_{1} X_{\mu_{v}}^{2}}{\left.\left.R_{2}[1-(1 \pm v) s]\right\}\left[\frac{R_{1}}{1-(1 \pm v) s}\right]^{2}+\left(X_{1}+X_{\mu_{v}}\right)^{2}\right\}}
$$

Maksimalnu vrednost, a stoga i naj5-tog harmonika, koji prema izrazu (5) važniji značaj, ima asinhroni moment iznosi:

$$
m_{5}=\left(\frac{I_{25}}{I_{2_{1}}}\right)^{2} \frac{s R_{1} X_{\mu_{5}}^{2}}{R_{2}(1-6 s)\left[\left(\frac{R_{1}}{1-6 s}\right)^{2}+\left(X_{1}+X_{\mu_{5}}\right)^{2}\right]}
$$

Maksimalna vrednost asinhronog momenta 5-tog harmonika ima se pri klizanju $\mathrm{s}=1 / 6$. U cilju procene vrednosti ovog asinhronog momenta smatraćemo da je $\mathrm{s}=1 / 6-\Delta \mathrm{s}$. Uzimajući u obzir da je $\Delta \mathrm{s}<<$ $1 / 6$, dobija se:

$$
m_{5 \max }=\left(\frac{I_{2_{5}}}{I_{2_{1}}}\right)^{2} \frac{X_{\mu_{5}}^{2}}{12 R_{2}\left(X_{\mu_{5}}+X_{1}\right)^{2}}
$$




\section{TEORIJSKA ANALIZA}

Iz izraza (5), može se videti da vrednost viših harmonijskih asinhronih momenata zavisi od vrste korišćenog frekventnog pretvarača i reda vremenskih viših harmonika, gde sa porastom reda harmonika, ova vrednost amplitude naglo opada. Za datu vrednost višeg harmonika, karakter i vrednost višeg harmonijskog asinhronog momenta zavisi od klizanja, t.j. od brzine obrtanja. Tako, 5-ti harmonik momenta je otporan (protivi se kretanju) u oblasti klizanja $0<\mathrm{s}<1 / 6$, t.j. za opseg brzina $5 / 6 n_{1}$ $<n<n_{1}$, dok je pogonski (podržava kretanje) u oblasti klizanja $1 / 6<\mathrm{s}<1$, t.j. za opseg brzina $0<n<5 / 6 n_{1}$. Sedmi harmonik asinhronog momenta je otporan pri svim brzinama. Jedanaesti harmonik asinhronog momenta je pogonski u oblasti $0<n<$ $11 / 12 n_{1}$, i otporan nakon toga, itd.

Prema izrazima (6) i (7), može se zaključiti da maksimalna vrednost 5-tog harmonika asinhronog momenta, koja se pojavljuje pri $s=1 / 6$, je mnogo viša od vrednosti drugih komponenti i da zato ima $\mathrm{i}$ visoku relativnu vrednost. Zapravo, ovo je izloženo u nekim ranije objavljenim istraživanjima. Medjutim, to nije tako, zbog sledećih razloga:

Prvo, reaktansa $X_{\mu_{5}}$ magnećenja petog harmonika je manja od odgovarajuće reaktanse osnovnog $X_{\mu_{1}}\left(X_{\mu_{5}}<X_{\mu_{1}}\right)$ harmonika, zbog zasićenja od stranje polja osnovnog harmonika [4].

Drugo, pri klizanju $\mathrm{s}=1 / 6$, 5-ti harmonik struje statora je jednak nuli $\left(I_{15}=0\right)$, zbog čega se peti harmonik fluksa naglo povećava, jer pri tome nema efekta prigušivanja od strane struje statora. Ovo dovodi do značajnog povećanja odgovarajuće ekvivalentne reaktanse, i direktno proizilazi iz ekvivalentnih šema za 5-ti harmonik pri $s=1 / 6$ i pri $s \neq 1 / 6$, kao što je prikazano na slikama 3 i 4.

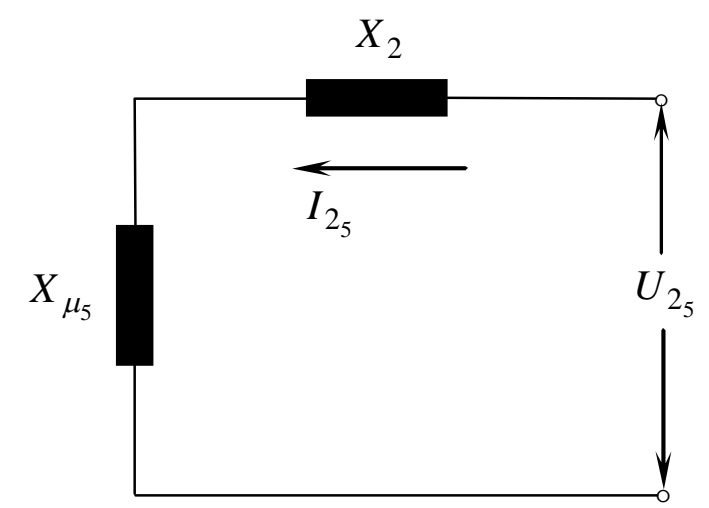

Sl. 3. Ekvivalentna šema asinhronog motora sa namotanim rotorom za 5-ti harmonik pri $s=1 / 6$

Sledstveno, 5-ti harmonik struje rotora pri $\mathrm{s}=1 / 6$ je manji od vrednosti pri $\mathrm{s} \neq 1 / 6$ jer je:

$$
\frac{I_{2_{5(s \approx 1 / 6)}}}{I_{2_{5(s \neq 1 / 6)}}}=\frac{X_{2}\left(1+c_{v_{5}}\right)}{c_{v_{5}}\left(X_{2}+X_{\mu_{5}}\right)}
$$




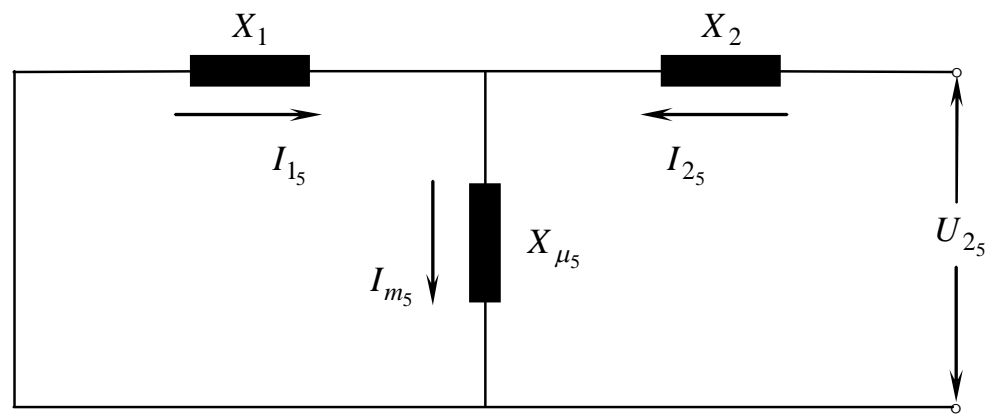

S1. 4. Ekvivalentna šema asinhronog motora sa namotanim rotorom za 5-ti harmonik pri $s \neq 1 / 6$

Gde je $\quad c_{v_{5}}=\frac{X_{1}+X_{\mu_{5}}}{X_{\mu_{5}}} \quad$ Hopkinsov koeficijent rasipanja za 5-ti harmonik koji za današnje asinhrone motore iznosi $c_{v_{5}}=1,15-1,3$, i reaktansa magnećenja 5tog harmonika je $X_{\mu_{5}}=(3-6) X_{2}$, t.j.:

$$
I_{25(s=1 / 6)}<I_{25(s \neq 1 / 6)}
$$

Kako 5-ti harmonik struje rotora ima značajan efekat na maksimalnu vrednost odgovarajućeg harmonijskog momenta (prema izrazu (8)), može se zaključiti da je ova vrednost mnogo manja od one koja bi bila da su zanemarene prethodno navedene činjenice

Harmonijski asinhroni moment 7-og harmonika nema maksimalnu vrednost u motornom radnom području (oblasti). Maksimalna vrednost 11-tog harmonika asinhronog momenta pojavljuje se pri klizanju $\mathrm{s}=1 / 12$ i ona je mnogo značajno niža od maksimalne vrednosti 5-tog harmonika asinhronog momenta.

\section{REZULTATI MERENJA I PRORAČUNI}

U cilju potvrđivanja izvedenih zaključaka i uvida u relativne vrednosti harmonijskih asinhronih momenata, eksperimentalna ispitivanja su izvršena na trofaznom asinhronom motoru sa namotanim rotorom, proizvodnje "Rade Končar", nazivne snage $6,4 \mathrm{~kW}$, čiji su parametri određeni u ranije izvršenim ogledima i koji iznose:

$$
\begin{array}{ll}
R_{1}=0,41 \Omega & R_{2}=0,60 \Omega \\
X_{1}=1,25 \Omega & X_{2}=1,25 \Omega \\
X_{\mu_{5}}=4,6 \Omega &
\end{array}
$$

Napajanje sa rotorske strane ostvareno je preko pretvarača tipa VF-6 proizvodnje General Electric, čiji izlaz talasni oblik napona prikazana na Slici 1. Merenje harmonijskog sadržaja struja statora i rotora izvršeno je pomoću elektronsko talasnog analizatora FRA-2d proizvodnje "Radiometar"Copenhagen. Dobijeni su sledeći rezultati:

$$
\begin{array}{ll}
\text { - pri klizanju } s \approx 1 / 6 & I_{2_{5}}=11 \% \\
\text { - pri klizanju } s=0,5 & I_{2_{5}}=28 \%
\end{array}
$$

Izračunavanjem prema izrazu (8) dobijena je maksimalna vrednost 5-tog harmonika asinhronog momenta:

$$
m_{5 \max }=0,81 \%
$$

Za motore srednjih i velikih snaga, relativna vrednost otpora statora je veoma mala, zbog čega se dobijaju veće vrednosti relativnog harmonijskog momenta $m$, koji međutim ne prelazi vrednost od 5\%. Vrednosti asinhronih momenata pri drugim klizanjima je manja od $1 \%$. 


\section{ZAKLJUČAK}

Harmonijski asinhroni momenti nastaju usled prisustva viših vremenskih harmonika u naponima i strujama asinhronog motora sa namotanim rotorom kod koga se brzina reguliše primenom frekventnih pretvarača $u$ kolo rotora. Proizvodi ih međusobno delovanje između viših vremenskih harmonika rotorskog fluksa i odgovarajućih harmonika struje statora. Teorijska analiza, izvršeni proračuni i eksperimentalni rezultati, koji su prikazani u ovom radu, pokazuju da su oni mali, uključujući maksimalnu vrednost 5-tog harmonika asinhronog momenta, koji nastaje pri klizanju $\mathrm{s}=1 / 6$ i kao takav nema značajan efekat na karakteristike motora.

Opisana metoda je razmatrala najnepovoljniji talasni oblik napona na izlazu iz frekventnog pretvarača, a to je pravougaoni talasni oblik napona, i pokazala da pri tome harmonijski asinhroni momenti nemaju značajan uticaj na vrednost momenta motora. Daljim oblikovanjem talasnog oblika napona može se potpuno eliminisati prisustvo asinhronih komponenti momenta.

\section{Dodatak}

$s \quad$ klizanje

$\Omega_{1} \quad$ brzina obrtnog polja statora

$f_{1}$ frekvencija mrežnog napajanja sa statorske strnae

$f_{2} \quad$ frekvencija sa rotorske strane

$v \quad$ redni broj harmonika

$\Omega_{1 v}$ brzina $v$-tog harmonika obrtnog polja statora

$S_{v} \quad$ klizanje $v$-tog harmonika

$R_{1} \quad$ otpornost statora po fazi

$R_{2} \quad$ otpornost rotora po fazi

$X_{1}$ reaktansa rasipanja statora po fazi

$X_{2}$ reaktansa rasipanja rotora po fazi

$X_{\mu} \quad$ reaktansa magnećenja za osnovnu frekvenciju

$X_{\mu \nu}$ reaktansa magnećenja za frekvenciju $v$-tog harmonika

$U_{1 v}$ napon statora po fazi za $v$-ti harmonik

$U_{2 v}$ napon rotora po fazi za $v$-ti harmonik
$I_{1}$ efektivna vrednost struje statora za osnovnu frekvenciju

$I_{2}$ efektivna vrednost struje rotora za osnovnu frekvenciju

$I_{1 v}$ efektivna vrednost struje statora za frekvenciju $v$-tog harmonika

$I_{2 v}$ efektivna vrednost struje rotora za frekvenciju $v$-tog harmonika

\section{LITERATURA}

[1] B. Jeftenić, M. Bebić, L. Ristić, S. Štatkić, "Design and Selection of Belt Conveying Equipment \& Systems," Chapter 2 in Book - Design and Selection of Bulk Material Handling Equipment and Systems: Mining, Mineral Processing, Port, Plant and Excavation Engineering. vol. I, Edited by Prof. Jayanta Bhattacharya, ISBN: 9788190904377, pages in Book: 254, pages in Chapter 2: 60, Publisher: Wide Publishing, Kolkata, West Bengal, India, 2011.

[2] A. Lavi, R.J. Polge: Induction motor speed control with static inverter in the rotor, IEEE Trans, PAS-85, No 1, 1987, pp. 76-84.

[3] M. Petrović: Ponašanje asinhrone mašne pri regulaciji pomoću tiristorske rekuperacije energije klizanja, Publikacije Elektrotehničkog fakulteta Univerziteta u Beogradu, serija Elektroenergetika, No 63, 1976.

[4] Dj. Vukić: Harmonic equivalent circuits of induction motor with semiconductor converter in the rotor, Review of research work at the Faculty of Agriculture, Vol. 38, 1993, pp. 119-127.

[5] Dj. Vukić, D. Stojanović i N. Mitrović: Uticaj viših harmonika na rad asinhronog motora sa poluprovodničkim pretvaračem u kolu rotora, Zbornik VIII Simpozijuma "Energetska elektronika", Novi Sad, septembar 1995, pp. 343-350. 